\title{
COMBUSTION WAVES
}

\author{
R. O. WEBER and S. D. WATT
}

(Received 22 July 1994; revised 21 April 1995)

\begin{abstract}
Finding critical phenomena in two-dimensional combustion is normally done numerically. By using a centre-manifold reduction, we can find a reduced equation in one dimension. Once we have found the reduced equation, it is simpler to find critical phenomena. We consider two different problems. One is spontaneous ignition. We compare our results with known critical parameters to give some validity to our reduction technique. We also look at a combustion model with three equilibrium states. For this model, the possible transitions can occur as travelling waves between the unstable to either of the stable equilibrium or from one stable to the other stable state. For the latter transition, the direction of the transition tells us whether we have an extinction or ignition wave. We find the critical parameters when the direction of the wave changes.
\end{abstract}

\section{Introduction}

Fisher [3] and Kolmogorov et al. [7] initiated the study of wave-like solutions to a single reaction-diffusion equation:

$$
u_{t}=u_{x x}+F(u)
$$

Suitable reaction functions, $F(u)$ must have multiple equilibria, with at least one equilibrium being stable. The two classes of functions which are most studied can be characterised as

$$
\begin{aligned}
& \text { Monostable ... e.g., Fisher, } F(u)=u(1-u), \\
& \text { Bistable... e.g., Nagumo, } F(u)=\left(u_{1}-u\right)\left(u_{2}-u\right)\left(u_{3}-u\right) .
\end{aligned}
$$

A monostable function has one unstable and one stable equilibrium and it is expected that waves would effect the transition from the unstable to the stable. A bistable

\footnotetext{
${ }^{1}$ Department of Mathematics, University College, Australian Defence Force Academy, Canberra ACT 2600, Australia

(C) Australian Mathematical Society, 1997, Serial-fee code 0334-2700/97
} 
function has two stable equilibria and one, unstable, intermediate equilibrium. It would now be expected that there could be three waves;

1. those that effect a transition from stable to stable (in either direction),

2. those that effect a transition from the unstable to the lower stable,

3. those that effect a transition from the unstable to the upper stable.

It is, in general, difficult to find critical phenomena in a two-dimensional problem. However, by using centre-manifold theory, a one-dimensional reduced approximation can be found. In this paper we wish to investigate the speed of wave-like solutions to a reaction-diffusion equation with two different reaction functions relevant to combustion, one monostable and the other bistable, via the reduced approximation.

\section{A look at two specific reaction functions}

Recent centre-manifold work [9] provides a way to realise the above classification of reaction-diffusion waves in the physically interesting combustion case. Two typical reaction functions in the combustion literature are (see, for example, Gray and Kordylewski, [5, 6])

$$
\begin{aligned}
& R_{1}(u)=\delta e^{u}, \\
& R_{2}(u)=\delta e^{u /(1+\epsilon u) .}
\end{aligned}
$$

As it stands, there are no equilibrium values for $u$ (so that $R_{i}(u)=0 ; i=1,2$ ). However, Watt et al. [9] (see Appendix A) show how a two-dimensional reactiondiffusion problem (with a general reaction and a domain $\mathbb{R} \times[0,1]$ ) can be systematically approximated by a one-dimensional equation

$$
T_{t}=T_{x x}+\overline{\psi R_{i}(\psi T)}-\lambda T
$$

where $T$ is a prescribed cross-bed average temperature and we define

$$
\bar{f}=\int_{0}^{1} f(x, y, t) d y,
$$

where

- $\psi^{\prime \prime}+\lambda \psi=0$,

- $\overline{\psi^{2}}=1$,

- $\lambda$ is the leading order decay rate,

- $\psi$ satisfies the boundary conditions of the two-dimensional problem. 
In the special case where the heat loss at the top and bottom is assumed to be small, $\psi=1$ and the reduced system becomes

$$
T_{t}=T_{x x}+R_{i}(T)-\lambda T,
$$

where this is the second-order approximation and $-\lambda T$ is a result of the heat loss on the boundaries.

The accuracy of this technique for the reaction function $\delta e^{u}$ is evaluated in Appendix $\mathrm{B}$, by using it to find the critical conditions for spontaneous ignition. The technique seems to give excellent approximate dynamics and can be systematically improved by calculating more terms in the series expansion.

For the combustion reaction functions, $R_{i}(u)$, this means that equilibria are now (conditionally) possible as

$$
\delta e^{u}-\lambda u=0
$$

or

$$
\delta e^{u /(1+\epsilon u)}-\lambda u=0
$$

have solutions for certain parameter ranges. Analytical formula for solutions do not exist. However sketches of $R_{i}(u)=\lambda u$ soon convince one that the solutions are as given in Figure 1a and $1 \mathrm{~b}$, for (9) and (10) respectively.

Figure la shows that $\delta e^{u}-\lambda u=0$ has two solutions for $\delta / \lambda<1 / e$, one solution when $\delta / \lambda=1 / e$ and no solutions for $\delta / \lambda>1 / e$. Stability of the solution branches (for $\delta / \lambda<1 / e$ ) is determined by making a small alteration to the equilibrium and studying its temporal evolution. It is soon apparent that the lower branch is stable and the upper branch is unstable as indicated on Figure 1a.

Figure $1 \mathrm{~b}$ shows that $\delta e^{u /(1+\epsilon u)}-\lambda u=0$ can have one, two or three solutions depending upon the value of $\delta / \lambda$, provided $0<\epsilon<0.25$. The exact values at the turning points, labelled $i$ and $e$, depend upon the chosen value of $\epsilon$.

The $u$ values at the turning points are found by requiring the slope of $R_{2}(u)-\lambda u$ to be zero at a solution of $R_{2}(u)-\lambda u=0$ :

$$
u_{c r}=\frac{(1-2 \epsilon) \pm \sqrt{1-4 \epsilon}}{2 \epsilon^{2}} \text {. }
$$

One can then find the value of $\delta / \lambda$ by substitution:

$$
\left(\frac{\delta}{\lambda}\right)_{c r}=u_{c r} e^{-u_{c r} /\left(1+\epsilon u_{c r}\right)}
$$

For example if $\epsilon=0.1$, the coordinates of the lower and upper turning points are $(\delta / \lambda, u)=(0.412,1.27)$ and $(0.011,78.73)$ respectively (see the table in Section 4 for more values). Studying the temporal evolution of small perturbations to the equilibria give the stability as indicated in Figure $1 \mathrm{~b}$. 

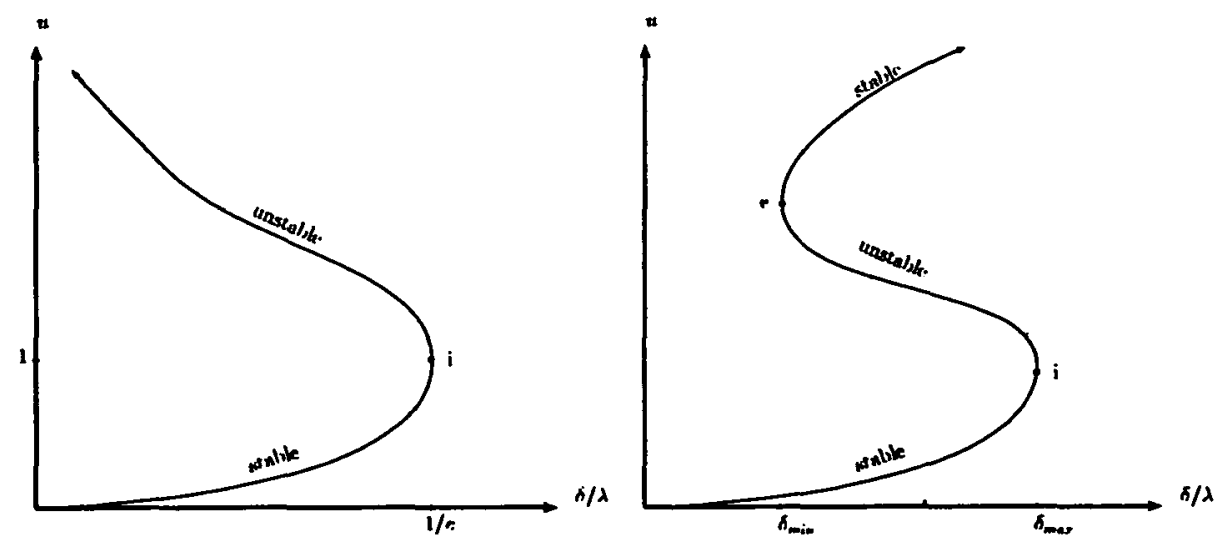

FIGURE 1. Sketches of the solutions of $R_{i}(u)=\lambda u$ for $i=1,2$ respectively.

\section{Combustion waves}

The analysis of the previous section shows that, for a given value of $\delta / \lambda, \delta e^{u}-\lambda u$ is a monostable reaction function, and $\delta e^{u /(1+\epsilon u)}-\lambda u$ is a bistable reaction function. This classification allows one to immediately see which wave solutions are possible in the combustion cases.

Namely, for $\delta e^{u}-\lambda u$, the only possible wave effects a transition from the unstable to the stable equilibrium. The speed of this wave is most easily found (as in all monostable cases) by linearising about the unstable equilibrium, whence

$$
c \geq 2 \sqrt{\delta e^{\mu}-\lambda}
$$

where $u$ is the equilibrium value, at a particular $\delta / \lambda$, attained on the unstable upper branch in Figure 1a. (Compare with Gray and Kordylewski [5] and Buonincontri and Hagstrom [1].)

For $\delta e^{u /(1+\epsilon u)}-\lambda u$, when $0<\epsilon<0.25$, there are three possible waves. Denoting the lower, intermediate and upper branches in Figure $1 \mathrm{~b}$ as $u_{1}, u_{2}, u_{3}$ respectively, 
these waves are categorised as effecting the transitions

$$
\begin{aligned}
& u_{1} \longleftrightarrow u_{3}, \\
& u_{2} \longrightarrow u_{1}, \\
& u_{2} \longrightarrow u_{3},
\end{aligned}
$$

where the arrows indicate the direction of the transition. (Compare with Gray and Kordylewski [6].)

In each case the wave speed is determined from the nonlinear eigenvalue problem

$$
\begin{aligned}
u^{\prime \prime}+c u^{\prime}+F(u) & =0, \\
u(-\infty)=u_{i}, \quad u(+\infty) & =u_{j}, \quad i \neq j \in\{1,2,3\} .
\end{aligned}
$$

Multiplying by $u^{\prime}$ and integrating from $-\infty$ to $+\infty$ gives an equation

$$
c=\frac{\int_{u_{j}}^{u_{i}} F(u) d u}{\int_{-\infty}^{\infty}\left(u^{\prime}\right)^{2} d z}
$$

for the wave speed. As the denominator is always positive (and non-zero), the numerator, that is, the area under the reaction function, is the crucial quantity for determining the speed of the reaction wave. In particular we notice that for the possibilities (14) the direction of the transitions between $u_{1}$ and $u_{3}$ are determined by the sign of $\int_{u_{1}}^{u_{3}} F(u) d u$. When this integral is positive, $u_{1} \rightarrow u_{3}$, and we refer to it as an ignition wave. When this integral is negative, $u_{3} \rightarrow u_{1}$, and we refer to it as an extinction wave.

Clearly, the value of this integral depends upon $\delta / \lambda$ which must be chosen for a particular application. Furthermore, a value for $\delta / \lambda$ (as a function of $\epsilon$ ) exists for which the integral is zero and no transition will occur. This border between transitions from $u_{1}$ to $u_{3}$ or from $u_{3}$ to $u_{1}$ will be examined in more detail in the next section.

An exact formula for the wave speed effecting transitions between $u_{1}$ and $u_{3}$ seems to be unavailable. However, we could try to avail ourselves of the analogy with the bistable equation, and in particular the Nagumo example, as the reaction function given in (3) leads to an exact travelling wave solution with speed

$$
c=\frac{1}{\sqrt{2}}\left(u_{1}-2 u_{2}+u_{3}\right) \text {. }
$$

Now, we check if the formula (18) is approximately correct for the combustion wave which effects a transition between $u_{1}$ and $u_{3}$. In particular we can see if the following hold:

$$
\begin{array}{rll}
u_{1} \rightarrow u_{3} & \text { if } & \left(u_{3}-u_{2}\right)>\left(u_{2}-u_{1}\right), \\
u_{3} \rightarrow u_{1} & \text { if } & \left(u_{3}-u_{2}\right)<\left(u_{2}-u_{1}\right), \\
\text { and } c=0 & \text { if } & \left(u_{3}+u_{1}\right)=2 u_{2} .
\end{array}
$$


From the table in the next section it is clear that (18) is not a good estimate of the speed in the combustion case.

Additionally, transitions from the intermediate unstable equilibrium $u_{2}$ to $u_{1}$ or $u_{3}$ should be amenable to analysis by linearisation. By using this method, which was successful for the monostable case, the speed for the Nagumo example in (3) is given by

$$
2 \sqrt{\left(u_{2}-u_{1}\right)\left(u_{3}-u_{2}\right)} \text {. }
$$

Similarly the speed for the reaction function $\delta e^{u /(1+\epsilon)}-\lambda u$ is found to be

$$
2 \sqrt{\frac{\delta e^{u_{2} /\left(1+\epsilon u_{2}\right)}}{\left(1+\epsilon u_{2}\right)^{2}}-\lambda} .
$$

Again we see that the Nagumo example is unsuited to deriving approximate wavespeed formulae.

\section{On the border of transitions}

Consider the reduced equation

$$
\frac{\partial u}{\partial t}=\frac{\partial^{2} u}{\partial x^{2}}+\delta e^{u /(1+\epsilon u)}-\lambda u
$$

where $\lambda$ is the heat loss coefficient, which we assume to be 1 .

This can have up to three equilibrium solutions, two stable and one unstable. We have discussed that the only physically-realisable transition is from one stable state to the other. While it would make the analysis complete if we could find the wave speed as a function of $\epsilon$ and $\delta$, it would be almost as beneficial to find when the direction of the transition changes.

As the speed is given by

$$
c=\frac{\int_{u_{1}}^{u_{3}} F(u) d u}{\int_{-\infty}^{+\infty}\left(u^{\prime}\right)^{2} d z},
$$

the change in direction occurs when $c=0$, or

$$
\int_{u_{1}}^{u_{3}} F(u) d u=0 .
$$

We have found these important parameters $\delta_{i m p}$ for selected values of $\epsilon$, as shown in the following table. 


\begin{tabular}{|c|c|c|c|c|c|c|c|}
\hline$\epsilon$ & $\delta_{\min }$ & $\delta_{\max }$ & $\delta_{i m p}$ & $u_{1}\left(\delta_{i m p}\right)$ & $u_{2}\left(\delta_{i m p}\right)$ & $u_{3}\left(\delta_{i m p}\right)$ & Area \\
\hline 0.00 & 0 & 0.36788 & - & 1 & 1 & 1 & - \\
0.05 & 0.000002 & 0.3878 & $2.3316 \times 10^{-6}$ & $2.3316 \times 10^{-6}$ & 233.524 & 582.20 & 8409.1 \\
0.10 & 0.011027 & 0.41153 & 0.011927 & 0.012071 & 51.022 & 125.78 & 330.97 \\
0.15 & 0.12830 & 0.44086 & 0.13619 & 0.159077 & 19.213 & 46.071 & 33.779 \\
0.20 & 0.35128 & 0.47953 & 0.36359 & 0.64250 & 8.6932 & 19.2316 & 3.30487 \\
0.25 & 0.54134 & 0.54134 & - & 4 & 4 & 4 & - \\
\hline
\end{tabular}

The "area" mentioned is that under each half of the bistable-reaction function when $\delta=\delta_{i m p}$. Naturally the total area vanishes for this value of $\delta$. Also, $\delta_{\min }$ and $\delta_{\max }$ are the $\delta$ values of the minimum and maximum turning points respectively.

Once we have these parameters, we can conclude that

1. if $\delta<\delta_{i m p}$ then $c<0$ and then $u_{3} \rightarrow u_{1}$. Thus we have an "extinction" wave.

2. if $\delta>\delta_{i m p}$ then $c>0$ and then $u_{1} \rightarrow u_{3}$. Thus we have an "ignition" wave.

We have also calculated the parameters at more frequent values for $\epsilon$. These are shown graphically in Figure 2. What we can see from this is that $\delta_{i m p}$ is always close to $\delta_{\min }$. This would suggest that given a travelling wave, that is $\delta$ such that $\delta_{\min }<\delta<\delta_{\max }$, it is most likely to be an "ignition" wave.

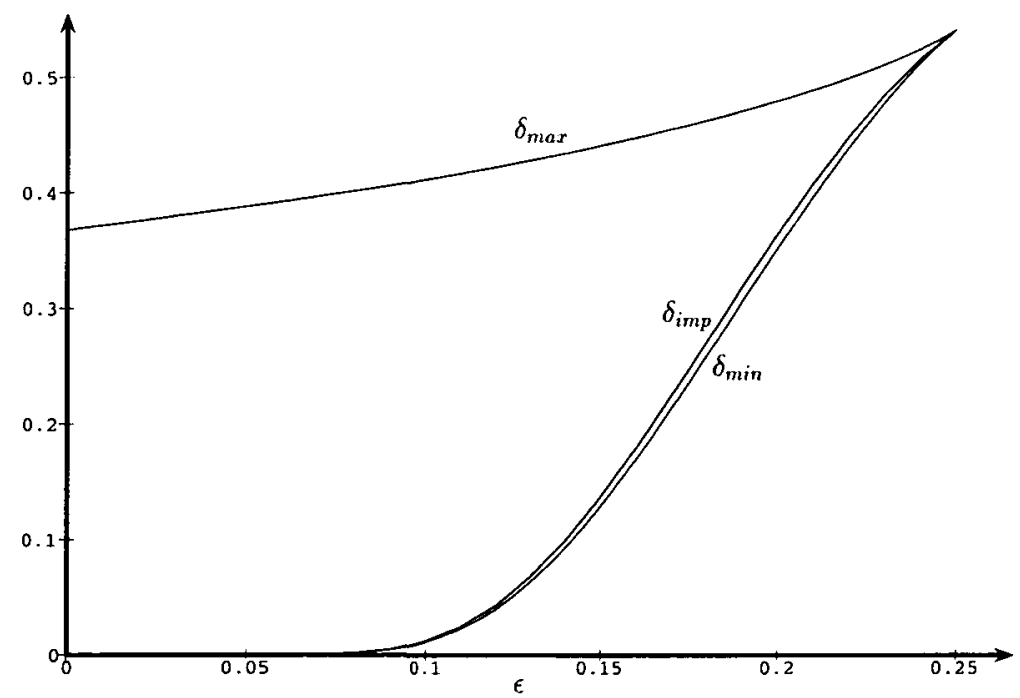

FIGURE 2. A graph of $\delta_{\min }, \delta_{i m p}$ and $\delta_{\max }$ as a function of $\epsilon$. 


\section{Conclusion}

We have analysed the waves which can be expected from a two-dimensional reaction-diffusion equation when the reaction is relevant to combustion. Our technique was to use an approximate one-dimensional model derived systematically using centre-manifold analysis. A range of realistic boundary conditions are suitable for this technique.

The two combustion functions considered yield approximate models with reaction functions which are monostable (one stable and one unstable equilibrium) or bistable (two stable and one unstable). It is then a straightforward matter to enumerate the possible waves which effect transitions between equilibria. In particular, the bistable function admits the possibility of a wave effecting a stable to stable transition, making it (potentially) a physical realisable reaction wave. The speed of this wave needs to be determined numerically. However we were able to understand its structure by studying the associated area under the reaction function. In particular, we give the parameter values which delineate between an extinction wave and an ignition wave. A complementary calculation shown in Appendix B demonstrates the impressive accuracy of the centre-manifold reduction.

Future work could focus on numerical determination of reaction waves with the function $\delta e^{u /(1+\epsilon u)}-\lambda u$.

\section{Appendix A： A summary of the centre manifold reduction}

In a previous paper [9], we have shown how to reduce a two-dimensional system with simple heat loss boundary conditions down to one-dimension. We can extend this to a system with more general boundary conditions.

Let us consider a two-dimensional equation

$$
\frac{\partial u}{\partial t}=\frac{\partial^{2} u}{\partial x^{2}}+\frac{\partial^{2} u}{\partial y^{2}}+R(u)
$$

with boundary conditions

$$
\begin{array}{ccc}
\alpha \frac{\partial u}{\partial y}+\beta u=0 & \text { at } & y=0, \\
\gamma \frac{\partial u}{\partial y}+\delta u=0 & \text { at } & y=1 .
\end{array}
$$

As in [9], we first assume that the horizontal diffusion and the reaction are small. Now considering the leading-order linear system, we have

$$
\frac{\partial u}{\partial t}=\frac{\partial^{2} u}{\partial y^{2}}
$$


satisfying the boundary conditions (28) and (29).

This equation has a solution

$$
u=\sum_{k=0}^{\infty} e^{\lambda_{k^{\prime}}}\left[A_{k} \cos \left(\nu_{k} y\right)+B_{k} \sin \left(\nu_{k} y\right)\right],
$$

where $\lambda_{k}=-v_{k}^{2}$, as are determined from (28) and (29).

The $\lambda_{k}$ 's are the eigenvalues corresponding to each eigenmode. Thus all modes decay exponentially, with the mode corresponding to $\lambda_{0}$ being of long-lasting importance. Let us base our system on this mode. As this mode has a decay rate of $-v_{0}^{2}$, which we will just call $-v^{2}$, our analysis will be an invariant-manifold analysis. This complicates our analysis, but we can get around this by modifying the original equation, (27), to

$$
\frac{\partial u}{\partial t}=\frac{\partial^{2} u}{\partial x^{2}}+\frac{\partial^{2} u}{\partial y^{2}}+(1-\epsilon) v^{2} u+R(u)
$$

and setting $\epsilon=1$ at the end. The eigenvalue spectrum changes to $\lambda_{k}=-v_{k}^{2}+v^{2}$. Now our leading-order decay rate is zero, so we can perform a centre-manifold analysis, which is simpler than an invariant-manifold analysis.

The leading-order approximation is

$$
u \sim A \cos (v y)+B \sin (v y)
$$

which we shall call $\psi$.

Assume that a centre manifold exists; that is, let $u(x, y, t)$ be some function of $T(x, t), u=V(T)$, where $T$ evolves according to $\partial T / \partial t=G(T)$.

We also must give a physical meaning to $T$. As in [9] we chose it to be a weighted cross-bed average,

$$
T=\overline{\psi u}=\int_{0}^{1} \psi(y) u(x, y, t) d y .
$$

Substituting this into (32), we get

$$
\frac{\partial V}{\partial T} G=\frac{\partial^{2} V}{\partial x^{2}}+\frac{\partial^{2} V}{\partial y^{2}}+(1-\epsilon) v^{2} V+R(V)
$$

This equation is as hard to solve as the original. However we can now use asymptotic techniques as described in Coullet and Spiegel [2] and Roberts [8]. That is, assuming asymptotic series for $V$ and $G$ of the form

$$
V \sim \sum_{n=0}^{\infty} v^{(n)} \quad G \sim \sum_{n=1}^{\infty} g^{(n)}
$$


we get a hierarchy of equations

$$
\frac{\partial^{2} v^{(n)}}{\partial y^{2}}+v^{2} v^{(n)}=\sum_{m=1}^{n} \frac{\partial v^{(n-m)}}{\partial T} g^{(m)}-\frac{\partial^{2}}{\partial x^{2}} v^{(n-1)}+\epsilon v^{2} v^{(n-1)}-R^{(n)}
$$

with subsidiary conditions

$$
\begin{array}{rrc}
\alpha \frac{\partial v^{(n)}}{\partial y}+\beta v^{(n)}=0 & \text { at } & y=0, \\
\gamma \frac{\partial v^{(n)}}{\partial y}+\delta v^{(n)}=0 & \text { at } & y=1, \\
\frac{\psi v^{(n)}}{2} & =\delta_{0, n} T,
\end{array}
$$

where $R^{(n)}$ is the $n^{\text {th }}$-order term in the Taylor series of the reaction

$$
R \sim \sum_{n=1}^{\infty} R^{(n)}
$$

At leading order, $n=0$, we assume that

$$
v^{(0)}=\psi T
$$

along with the normalisation condition $\overline{\psi^{2}}=1$.

At the next order, $n=1$, we have

$$
\frac{\partial^{2} v^{(1)}}{\partial y^{2}}+v^{2} v^{(1)}=\psi g^{(1)}-\frac{\partial^{2} T}{\partial x^{2}} \psi+\epsilon \psi v^{2} T-R(\psi T)
$$

Taking the weighted average of this with respect to $\psi$ yields

$$
0=g^{(1)}-\frac{\partial^{2} T}{\partial x^{2}}+\epsilon \nu^{2} T-\overline{\psi R(\psi T)}
$$

Thus to a first approximation, we have

$$
\frac{\partial T}{\partial t}=\frac{\partial^{2} T}{\partial x^{2}}+\overline{\psi R(\psi T)}-v^{2} T
$$

This equation is the foundation for our analysis of critical phenomena in this paper. 


\section{Appendix B: Spontaneous ignition}

Consider the system

$$
\frac{\partial u}{\partial t}=\frac{\partial^{2} u}{\partial x^{2}}+\frac{\partial^{2} u}{\partial y^{2}}+\delta e^{u} .
$$

By using centre-manifold theory, as described in Appendix A, we get a reduced system

$$
\frac{\partial T}{\partial t}=\frac{\partial^{2} T}{\partial x^{2}}+\delta \overline{\psi e^{\psi T}}-v^{2} T .
$$

The solutions to this will blow up exponentially if the reaction is strictly positive. So we must find a critical $\delta, \delta_{c r}$, such that the reaction function just touches the axis then turns up, as shown in Figure 3. Thus we need

$$
\begin{aligned}
& R\left(\delta_{c r}, T_{c r}\right)=\delta_{c r} \overline{\psi e^{\psi T_{c r}}}-v^{2} T_{c r}=0, \\
& \text { and } \frac{\partial R}{\partial T}\left(\delta_{c r}, T_{c r}\right)=\delta_{c r} \overline{\psi^{2} e^{\psi T_{c r}}}-\nu^{2}=0 .
\end{aligned}
$$

From these two we eliminate $v^{2}$ to find

$$
T_{c r} \overline{\psi^{2} e^{\psi T_{c r}}}-\overline{\psi e^{\psi T_{c r}}}=0
$$

where

$$
\delta_{c r}=\frac{v^{2} T_{c r}}{\overline{\psi e^{\psi T_{c r}}}} .
$$

From (50) and (51), we have found critical $\delta$ 's for a few boundary conditions, as shown below.

\begin{tabular}{|c|c|c|c|c|}
\hline Boundary & Conditions & $\delta_{c r}$ & $\delta_{c r}^{\text {known }}[4]$ & Difference \\
\hline$u^{\prime}(0)=0$ & $u(1)=0$ & 0.8773 & 0.8785 & $0.1 \%$ \\
$u(0)=0$ & $u^{\prime}(1)=0$ & 0.8773 & 0.8785 & $0.1 \%$ \\
$u(0)=0$ & $u(1)=0$ & 3.509 & 3.514 & $0.1 \%$ \\
\hline
\end{tabular}

Note here that the first and second critical $\delta$ is the same. This is because the problems are complementary to each other. Also the third critical $\delta$ is four times the first. This is because the problems are identical except that the third one is in a region half as small. Also note that we took the centre-manifold analysis to the next order and found numerically, the critical $\delta \approx 0.878517$ or a difference of about $0.006 \%$ of the known value [4]. 


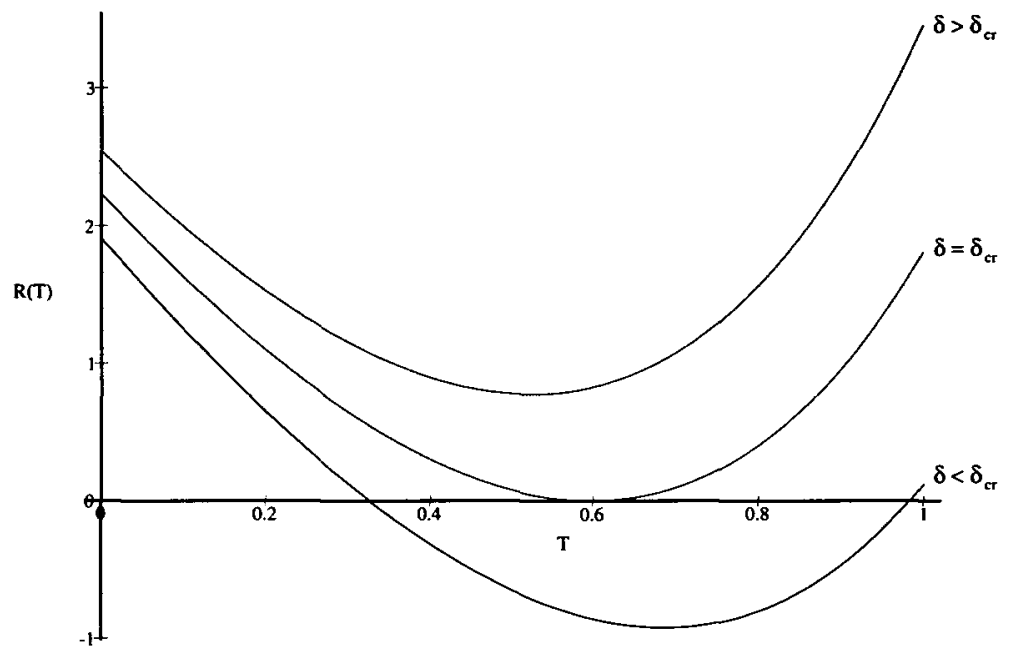

FIGURE 3. A sketch of a typical reaction function for spontaneous combustion

As a slight variation, we consider the problem in polar coordinates

$$
\frac{\partial u}{\partial t}=\frac{\partial^{2} u}{\partial r^{2}}+\frac{1}{r} \frac{\partial u}{\partial r}+\frac{1}{r^{2}} \frac{\partial^{2} u}{\partial \theta^{2}}+\delta e^{u}
$$

with

$$
\begin{aligned}
& \frac{\partial u}{\partial r}=0 \quad \text { at } \quad r=0, \\
& u=0 \quad \text { at } \quad r=1 .
\end{aligned}
$$

This reduces to

$$
\frac{\partial T}{\partial t}=\overline{\frac{1}{r} J_{0}(\nu r)^{2}} \frac{\partial^{2} T}{\partial \theta^{2}}+\overline{\delta r c_{1} J_{0}(v r) e^{c_{1} J_{0}(\nu r) T}}-v^{2} T,
$$

where $J_{0}$ is the zeroth order Bessel function, $v$ is the first zero of $J_{0}$ and $c_{1}=\frac{2 v^{2}}{\left(b^{2}+v^{2}\right) J_{0}(v)}$. For these reduced equations the critical $\delta$ is 1.9917 , compared to 2 [4], a difference of about $0.42 \%$.

From these examples it seems the centre-manifold reduction gives excellent estimates of the bifurcation phenomena. 


\section{Acknowledgement}

R.O. Weber thanks the School of Computing and Mathematics at Deakin University, and particularly Professor Peter Kloeden, for their hospitality while this work was completed. We also thank Professor Tony Roberts for his helpful discussions.

\section{References}

[1] S. Buonincontri and T. Hagstrom, "Multidimensional travelling wave solutions to reaction-diffusion equations", IMA J. Appl. Math. 43 (1989) 261-271.

[2] P. Coullet and E. A. Spiegel, "Amplitude equations for systems with competing instabilities", SIAM J. Appl. Math. 43 (1983) 776-821.

[3] R. A. Fisher, "The wave of advance of advantageous genes", Ann. Eugenics 7 (1937) 353-369.

[4] D. A. Frank-Kamenetskii, Diffusion and heat transfer in chemical kinetics, second ed. (Plenum Press, N.Y., 1969).

[5] P. Gray and W. Kordylewski, "Standing waves in exothermic systems", Proc. R. Soc. Lond. A398 (1985) 281-288.

[6] P. Gray and W. Kordylewski, "Travelling waves in exothermic systems", Proc. R. Soc. Lond. A416 (1988) 103-113.

[7] A. Kolmogorov, I. Petrovsky and N. Piscounoff, "Study of the diffusion equation with growth of the quantity of matter and its application to a biology problem", in Dynamics of Curved Fronts (ed. P. Pelcé), (Academic Press, 1988).

[8] A. J. Roberts, "Simple examples of the derivation of amplitude equations for systems of equations possessing bifurcations", J. Austral. Math. Soc. Ser. B 27 (1985) 48-65.

[9] S. D. Watt, A. J. Roberts and R. O. Weber, "Dimensional reduction of a bushfire model", Math. Comp. Mod. 21 (1995) 79-83. 